\title{
Using a Combination of Micro-Computed Tomography, CAD and 3D Printing Techniques to Reconstruct Incomplete 19th-Century Cantonese Chess Pieces
}

\author{
S. D. LAYCOCK, School of Computing Sciences, University of East Anglia, NR4 7TJ \\ G. D. BELL, East Anglian Radiography Research, Modelling and 3-D printing group, School of Science, \\ Technology and Health, University Campus Suffolk, Ipswich, IP4 1QJ, 2UK \\ N. CORPS, Bruker UK, Banner Lane, Coventry CV4 9GH \\ D. B. MORTIMORE, Newbourne Solutions Ltd, Newbourne, Woodbridge, IP12 4NR, UK \\ G. COX, East Anglian Radiography Research, Modelling and 3-D printing group, School of Science, \\ Technology and Health -Suffolk, Ipswich, IP4 1QJ, 2UK \\ S. MAY, School of Computing Sciences, University of East Anglia, NR4 7TJ \\ I. FINKEL, The British Museum, Great Russell Street, London WC1B 3DG
}

\begin{abstract}
As scanning technologies improve new approaches to digitizing cultural heritage artefacts emerge. The use of micro-computed tomography (micro-CT) presents an interesting approach as it is capable of high-resolution scans of not just the surface of the artifact but also the intricate internal structures and volumes leading to more complete digitization than is possible with traditional surface laser scanning techniques. In this work, we investigate the potential to digitize heritage artifacts using micro-CT and focus on the methods of interacting with the datasets to create digital restorations of broken and incomplete pieces before printing physical replicas using 3D printing technology. We visualize the virtual models with clear identification of the new portions added by a digital artist with reference to existing pieces and archives.
\end{abstract}

Categories and Subject Descriptors: I.4.1 [Computing Methodologies]: Image Processing and Computer Vision—Digitization and image capture; I.3.5 [Computing Methodologies]: Computer Graphics-Computational geometry and object modeling

Additional Key Words and Phrases: Digitization, 3D printing, reconstruction, 3D modelling

ACM Reference Format:

S. D. Laycock, G. D. Bell, N. Corps, D. B. Mortimore, G. Cox, S. May, and I. Finkel. 2015. Using a combination of micro-computed tomography, cad and 3D printing techniques to reconstruct incomplete 19th-century Cantonese chess pieces. ACM J. Comput. Cult. Herit. 7, 4, Article 25 (February 2015), 6 pages.

DOI: http://dx.doi.org/10.1145/2629682

\section{INTRODUCTION}

In our previous article [Laycock et al. 2012], we reported the combined use of x-ray micro-CT and $3 \mathrm{D}$ printing technology to study and then print a 19th-century Cantonese ivory chess piece with an

Authors' addresses: S. D. Laycockl (Corresponding author); email: S.laycock@uea.ac.uk; G. D. Bell; email: duncan@ fishersideas.co.uk; N. Corps; email: nickcorps@yahoo.co.uk; D. B. Mortimore; email: david.mortimore@virgin.net; G. Cox; email: gcoxanimation@gmail.com; S. May; email: s.may@uea.ac.uk; I. Finkel; IFinkel@britishmuseum.org.

Permission to make digital or hard copies of part or all of this work for personal or classroom use is granted without fee provided that copies are not made or distributed for profit or commercial advantage and that copies show this notice on the first page or initial screen of a display along with the full citation. Copyrights for components of this work owned by others than ACM must be honored. Abstracting with credit is permitted. To copy otherwise, to republish, to post on servers, to redistribute to lists, or to use any component of this work in other works requires prior specific permission and/or a fee. Permissions may be requested from Publications Dept., ACM, Inc., 2 Penn Plaza, Suite 701, New York, NY 10121-0701 USA, fax +1 (212) 869-0481, or permissions@acm.org.

(c) 2015 ACM 1556-4673/2015/02-ART25 $\$ 15.00$

DOI: http://dx.doi.org/10.1145/2629682

ACM Journal on Computing and Cultural Heritage, Vol. 7, No. 4, Article 25, Publication date: February 2015. 
intricate internal structure due to its puzzle-ball base. Figure 1 shows the original Cantonese bishop used in our previous study. Large numbers of Cantonese ivory chess sets were produced in Canton between 1790 and 1910 for the European export market [Murray 1913; Hammond 1950; Wichmann and Wichmann 1964; Bell 1969, 1988; Finkel 2007]. Unfortunately, as these pieces are delicate and made up of several individual parts, as shown with the disassembled piece in Figure 1, many sets are now incomplete due to breakages and sometimes losses of some of the individual pieces or their constituent parts. In this article, we show how we can create complete Cantonese chess pieces based on utilizing micro-CT data of complete pieces, as shown in our digitization of a complete Cantonese ivory chess piece [Laycock et al. 2012]. Since the pieces have common elements, it is possible to use the accurate scans of one piece to aid in the reconstruction of another damaged or incomplete piece. This article describes the chess pieces used, the methods employed, and the results obtained. Where data is introduced such as for completing parts no longer in existence, images are provided to clearly identify these portions and references to sources of images of the pieces used by the digital artist are included.

\section{RELATED WORK}

Scanning of human anatomy using CT is now commonplace, and these datasets have been used increasingly in the production of 3D prints to aid surgical [Esses et al. 2011] and interventional radiotherapy planning [Tam et al. 2013], as well as human physiology studies [Giesel et al. 2009]. Abel et al. 2011] summarized the possible role of x-ray micro-CT in the study, preservation and dissemination of knowledge relating to various artifacts. They compared and contrasted x-ray micro-CT and other methods such as photography, serial sectioning, and laser surface scanning techniques and mentioned the possibility of linking the micro-CT imaging 3D data to 3D printer technology. Pitzalis et al. [2008] utilize CT to capture details present in the mineral structure of a cylinder seal. They also used optical microtomography to obtain surface details with a precision of up to $0.1 \mu \mathrm{m}$. In our previous article [Laycock et al. 2012], we did exactly that using a single complete 19th-century Cantonese ivory chess piece with an intricate internal structure. Guidi et al. [2014] investigated the importance of expert feedback during reconstruction to avoid misleading details.

\section{DESCRIPTION OF THE IVORY CHESS PIECES AND THEIR PROVENANCE}

The artifacts used in this work had been in the possession of one of the authors' (G.D. Bell) father, the late R.C. Bell [Bell 1969, 1988]. In this work, we used a complete Cantonese ivory bishop shown in Figure 1, the partially complete rook represented by an elephant with tower shown in Figure 2(a), and a partially complete Mongolian archer representing the knight, which is shown in Figure 2(b). The rook was missing its puzzle-ball base, and the knight was missing its base in addition to many intricate parts including parts of the arrows, the horse's legs, and a base for the horse to stand on. The provenance of these three Cantonese chess pieces was worked out from looking at descriptive text, figures, and photographs in appropriate books [Murray 1913; Hammond 1950; Wichmann and Wichmann 1964; Bell 1969, 1988; Finkel 2007]. On this basis, the three pieces were identified as being parts of a bishop, rook, and knight. All three pieces were ivory colored; however, it is typical for opposing pieces to be stained red. The heights of the incomplete rook and knight ivory pieces were $7.12 \mathrm{~cm}$ and $4.41 \mathrm{~cm}$, respectively, while the original complete bishop was $9.82 \mathrm{~cm}$ high [Laycock et al. 2012].

\section{DIGITIZING THE CANTONESE CHESS PIECES, SEGMENTING THE PUZZLE BALL, AND CREATING THE 3D PRINTS OF THE RECONSTRUCTED STL FILES}

The two incomplete chess pieces were scanned by one of the authors (N. Corps) using a SkyScan 1173 in vivo scanner. This scanner currently retails for approximately $£ 250 \mathrm{~K}$, although it is possible to use 


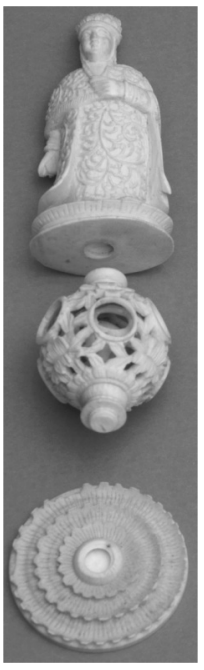

Fig. 1. The original Cantonese bishop chess piece shown in its three parts.

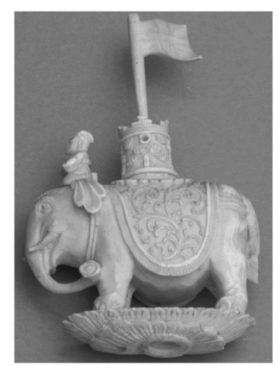

(a)

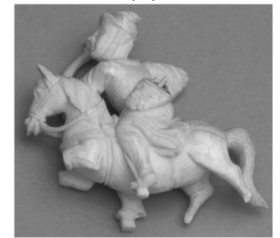

(b)

Fig. 2. Images of incomplete Cantonese ivory chess pieces. (a) Rook missing its puzzle-ball base. (b) Knight missing its base, arrows, and horse's legs with rock.

one at a rate of $£ 60$ per hour. A scanning resolution of $8.7 \mu \mathrm{m}$ was used to permit good visualization of the tooling marks and internal structure of the ivory pieces. This system uses the more conventional geometry (for micro-CT systems) of a fixed camera and source with a horizontal x-ray beam path, with the object being rotated around its vertical axis between them. As with the Cantonese bishop [Laycock et al. 2012], a single field of view was insufficient to scan the entire object. In this case, two scans were made in sequence with the object vertically displaced between the two scans. The overlap of the two resulting scan datasets was calculated automatically at the time of reconstruction and a single fused dataset was output. The total scan and reconstruction time for this object was 3 hours.

In order to produce manageable file sizes for the printing process, the original axial slice datasets were down-sampled uniformly using the Tomomask software (http://www.tomomask.com) to 2,000 pixels x 2,000 pixels x 5,297 slices with an isotropic spacing between pixels and slices of $17.3 \mu \mathrm{m}$. These TIFF data stacks could then be exported into the Disect viewing software (http://www.disectsystems. com). Images from Disect are shown in Figures 3(a) and 3(b). For each model scanned, it was possible to convert the stacks of axial data slices into an STL format using Tomomask in approximately 30 minutes. The models were scaled to the correct size according to the scanning resolution. In the case of the rook, the screw hole in the bottom of the upper base was filled in. The rook's upper base could then be joined to the puzzle ball and lower base data originally acquired from the original intact bishop [Laycock et al. 2012] using a modeling package. For this work, Maya (http://www.autodesk.com) was used, although alternative 3D modeling packages could be used that are capable of file conversion and editing geometry. The resulting STL (now complete with its own puzzle balls and base) was then sent to Shapeways Ltd. (http://www.shapeways.com) and printed in a polished white strong and flexible nylon material (Figure 4(c)).

In the case of the Mongolian mounted archer piece, the STL (made in Tomomask) was also first imported into Maya. This was used to convert the Mongolian mounted archer and the other component STL files into the OBJ file format. Most 3D modeling packages support file format conversion and so an alternative package (such as 3DS Max or Blender) could have been used. The "broken" sections 


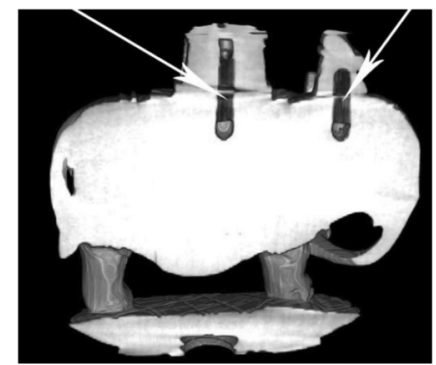

(a)

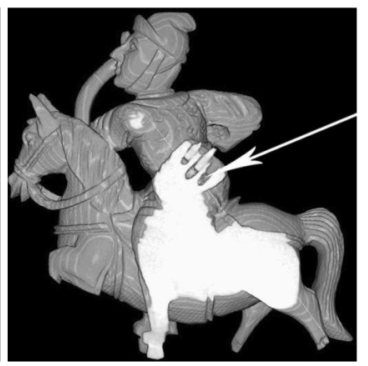

(b)

Fig. 3. Images of the incomplete Cantonese rook and knight taken from the axial slices of the micro-CT scan and viewed in Disect software. (a) shows that the elephant and its upper base are carved from a single piece of ivory but the tower on its back, its flag, and the mahout are all made separately and are attached with small wooden pegs. (b) The knight is missing its legs and parts of the arrows as indicated by the white arrow.

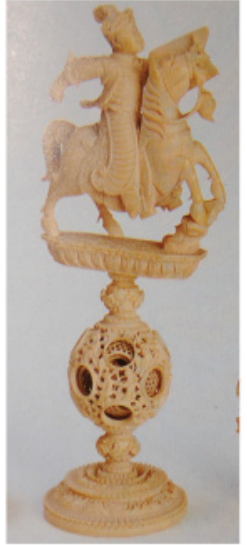

(a)

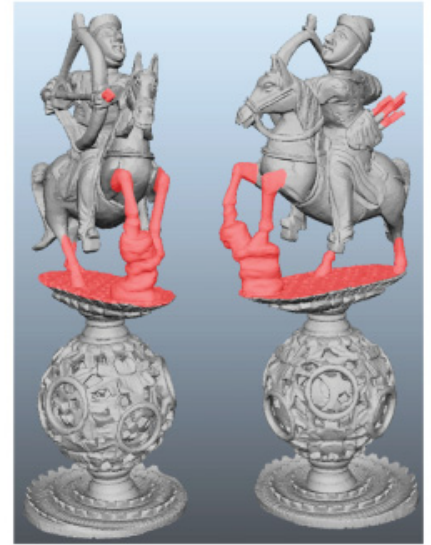

(b)

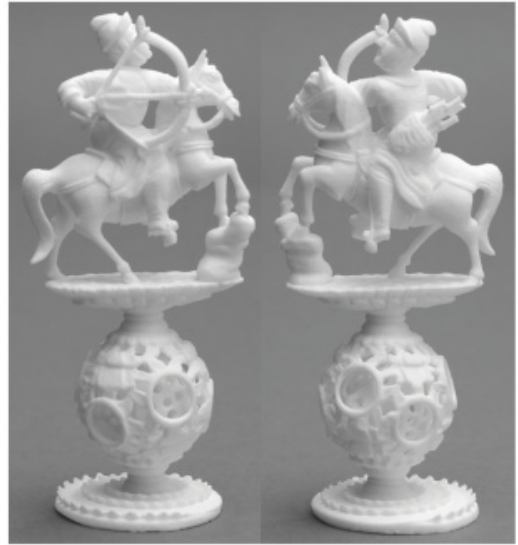

(c)

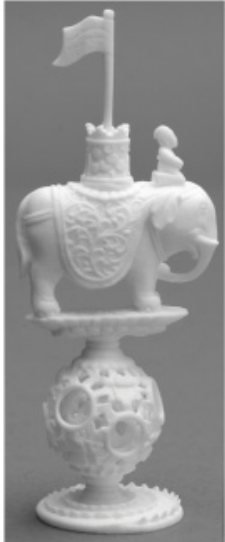

d

Fig. 4. A photograph of a complete knight [Bell 1988] is presented in (a) with permission of Penguin Books. This image was used to guide the reconstruction of the missing parts. A rendering from ZBrush showing the reconstruction, with the red parts indicating the additions, is presented in (b). The remaining parts were acquired from the micro-CT data. (c) Images of 3D prints of the reconstructed knight. (d) Image of the 3D print of the reconstructed rook. These models were completed using the puzzle-ball base from the Cantonese bishop.

could then be imported into ZBrush (http://www.pixologic.com), a digital sculpting and painting program. ZBrush was used to digitally reconstruct the missing sections in a manner that mirrored the original carving process as it has some advanced mesh editing features. The reconstruction process took approximately 12 hours with the aid of reference images of the complete piece illustrated in Bell [1988] and shown in Figure 4(a). The original carvers were extremely skilled, although subtle geometric differences in pieces can occur. Once the digital reconstruction was created, the authors discussed the resulting images with Dr C. Barclay, curator of the Oriental Museum at Durham University. The Oriental Museum currently holds a collection of Cantonese chesspeices left to them by the late R.C. Bell. The areas that were reconstructed were as follows: the horse's legs, a support for them, the missing tip on the arrow in the archer's bow, and the quiver of a number of arrows. Then, using Maya, as with the rook, the now completed upper piece of the archer was again joined to the puzzle ball and base data acquired from the original Cantonese piece [Laycock et al. 2012]. Figure 4(b) shows the mounted 
archer where the sections shaded red indicate the parts created by the digital artist in ZBrush. The resulting model was converted back to an STL (now complete with its own puzzle balls and base) and was similarly sent to Shapeways Ltd. (http://www.shapeways.com) and printed in a polished white strong and flexible nylon material (see Figure 4(c)). Finally, a mirror image of the Mongolian mounted archer was produced by mirroring the geometry in a 3D modeling package. Both the normal mounted archer and its mirror image were then printed out in a powder-based material using a ZCorps $4503 \mathrm{D}$ printer in approximately 2 hours. The printer has an accuracy of $0.13 \mathrm{~mm}$ and currently retails at approximately $£ 30 \mathrm{~K}$. However, even more accurate printers could lead to better-quality reproductions in the future in a wide range of materials. 3D printing services provided by companies such as Shapeways Ltd. are particularly appealing.

\section{RESULTS AND DISCUSSION}

We have shown it is possible to reconstruct whole Cantonese chess pieces with moving puzzle balls by performing x-ray micro-CT scans of damaged or incomplete pieces, producing highly detailed STL files, joining them to data previously acquired from a complete piece [Laycock et al. 2012], and then printing the resultant STL on a 3D printer. Unlike the original bishop chess piece [Laycock et al. 2012], we found no internal defects in the ivory of the new pieces shown in Figures 2(a) and 2(b). However, as can be seen in the 3D cut plane views shown in Figures 3(a) and 3(b), the micro-CT scans revealed the fact that although the chess figures themselves were made from a single piece of ivory, the tower, flag, and mahout of the rook and the arrow tip in the knight's bow and the arrows in his quiver were made separately. This gives valuable insight into the methods used by the original Cantonese carver.

The bishop is dressed as a mandarin in the style of the Manchu court (1644-1911). The knight appears to be a mounted archer of a Mongolian warrior rather than a Chinese horseman. This is not unexpected as many of the Cantonese ivory puzzle-ball chess sets made between 1790 and 1910 had one side (often the red pieces) made as Chinese officials and military personnel, while the other (often the white side) were carved either as Western or alternatively Mongolian figures. With a normal modern European set such as those of the Staunton type [Bermingham 1935], it would only be necessary to have six complete and undamaged pieces, that is, one pawn, one rook, one knight, one bishop, one queen, and one king chessman, to be able to reconstruct an entire set. Half would be of a darker color and the other half would be of a lighter color to represent black and white. In contrast, to make a complete Cantonese set, it would be necessary to have 12 pieces to make a complete set since the two sides are not only different colors (usually white and red) but also depicted as Chinese figures on one side versus either Western or Mongolian figures on the other.

In the case of the Mongolian mounted archer, in order to make the horse's legs strong enough, the front legs were given a support similar to those illustrated elsewhere (see Figure 238 on page 173 of Bell [1988]). With the ZBrush software, it was also possible to put some missing arrows into the mounted archer's quiver and replace the missing tip one the arrow in his bow (see Figure 4(c)). The techniques we have shown could easily be adapted by museums for repairing damaged artifacts or making accurate and pleasing reproduction models for sale or education purposes.

\section{REFERENCES}

R. L. Abel, S. Parfitt, N. Ashton, S. G. Lewis, B. Scott, and C. Stringer. 2011. Digital preservation and dissemination of ancient lithic technology with modern micro-ct. Computers and Graphics 35, 4 (2011), 878-884.

R. C. Bell. 1969. Board and Table Games from Many Civilisations. Oxford University Press.

R. C. Bell. 1988. Board and Table Games for All the Family. Penguin Books.

E. H. Bermingham. 1935. Staunton's Chess-Player Handbook. Bell \& Sons.

S. J. Esses, P. Berman, A. I. Bloom, and J. Sosna. 2011. Applications of physical 3D models derived from MDCT data and created by rapid prototyping. AJR 196, 6 (2011), 683-688. 
I. L. Finkel. 2007. Ancient Board Games in Perspective. British Museum Press.

F. L. Giesel, A. Mehndiratta, H. von Tengg-Kobligh, A. Schaeffer, K. Teh, H. U. Kauczor, E. J. van Beek, and J. M. Wild. 2009. Rapid prototyping raw models on the basis of high resolution tomography lung data for respiratory flow dynamics. Academic Radiology 16, 4 (2009), 495-498.

G. Guidi, M. Russo, and D. Angheleddu. 2014. 3D survey and virtual reconstruction of archeological sites. Digital Applications in Archaeology and Cultural Heritage 1, 2 (2014), 55-69.

A. Hammond. 1950. The Book of Chessman. Barker.

S. D. Laycock, G. D. Bell, D. B. Mortimore, M. K. Greco, N. Corps, and I. Finkel. 2012. Combining x-ray micro-ct technology and $3 \mathrm{~d}$ printing for the digital preservation of a 19th century cantonese chess piece with intricate internal structure. ACM Journal on Computing and Cultural Heritage 5, 4 (2012), 13.1-13.7.

H. J. R. Murray. 1913. History of Chess. Oxford University Press.

D. Pitzalis, P. Cignoni, M. Menu, and G. Aitken. 2008. 3D enhanced model from multiple data sources for the analysis of the cylinder seal of ibni-sharrum. VAST 35, 4 (2008), 79-84.

M. D. B. S. Tam, S. D. Laycock, D. Jayne, J. Babar, and B. Noble. 2013. 3-D printouts of the tracheobronchial tree generated from CT images as an aid to management in a case of tracheobronchial chondromalacia caused by relapsing polychondritis. Journal of Radiology Case Reports 7, 8 (2013), 34-43.

H. Wichmann and S. Wichmann. 1964. The Story of Chess-Pieces from Antiquity to Modern Times. Paul Hamlyn.

Received November 2013; revised May 2014; accepted May 2014 